\title{
Relaxation of selective constraints on avian mitochondrial DNA following the degeneration of flight ability
}

\author{
Yong-Yi Shen, ${ }^{1,2,3}$ Peng Shi, ${ }^{1,4}$ Yan-Bo Sun, ${ }^{1,2,3}$ and Ya-Ping Zhang ${ }^{1,2,4}$ \\ ${ }^{1}$ State Key Laboratory of Genetic Resources and Evolution, Kunming Institute of Zoology, The Chinese Academy of Sciences, Kunming \\ 650223, China; ${ }^{2}$ Laboratory for Conservation and Utilization of Bio-Resources, Yunnan University, Kunming 650091, China; \\ ${ }^{3}$ Graduate School of the Chinese Academy of Sciences, Beijing 100039, China
}

\begin{abstract}
The evolution of flight is the most important feature of birds, and this ability has helped them become one of the most successful groups of vertebrates. However, some species have independently lost their ability to fly. The degeneration of flight ability is a long process, and some species remain transitional locomotive models. Most of the energy required for locomotion is supplied by mitochondria via oxidative phosphorylation. Thus, rapidly flying birds should require a more energy efficient metabolism than weakly flying or flightless species. Therefore, we speculated that evolutionary constraints acted on the mtDNA of birds with different locomotive abilities. To test this hypothesis, we compared 76 complete avian mitochondrial genomes. Weakly flying and flightless birds, compared to rapidly flying birds, accumulated more nonsynonymous nucleotide substitutions relative to synonymous substitutions. Even after controlling for mutation rate, this trend remained significant. This finding was further tested for its generality by examining 214 complete mammalian mitochondrial genomes. The same as birds, a negative correlation was also found for the $K_{\mathrm{a}} / K_{\mathrm{s}}$ ratio and locomotive speed. Our results demonstrated that, in addition to the previously described role for effective population size, functional constraints due to locomotion play an important role in the evolution of mtDNA.
\end{abstract}

[Supplemental material is available online at http://www.genome.org. The sequence data from this study have been submitted to GenBank (http://www.ncbi.nIm.nih.gov/Genbank/) under accession nos. EU165706, EU165707, and EU417810-EU417812.]

Birds evolved from a single common ancestor (Sibley and Ahlquist 1990; Chiappe 2007). Flight is the most notable feature distinguishing them from all other vertebrates, and is their primary means of locomotion for breeding, feeding, and predator avoidance. Although this ability has helped them become one of the most successful taxa, about 60 extant (and many extinct) species have on several instances, secondarily and independently, lost their ability to fly (e.g., kiwi, penguins, and ratites) (Roots 2006). The degeneration of flight ability is a long process, and some species remain transitional locomotive models. Whereas extensive research evaluates the morphology of flight (Withers 1981; Berg and Rayner 1995), few genetic studies have addressed this subject.

Because mitochondria provide up to $95 \%$ of eukaryotic cell energy, we speculated that they must play an important role in the evolution of locomotive ability in birds. For the past $20 \mathrm{yr}$, mtDNA has commonly been considered to be a neutral marker and widely applied in evolutionary and conservation studies (Ballard and Whitlock 2004; Rand 2008). The role of selection in mtDNA evolution attracted little attention until recently (Mishmar et al. 2003; Elson et al. 2004; Ruiz-Pesini et al. 2004; Sun et al. 2007; Castoe et al. 2008; da Fonseca et al. 2008). Because mitochondria generate heat, climate (thermal environments) was suggested to be an important factor influencing the evolution of human mtDNA (Mishmar et al. 2003; Ruiz-Pesini et al. 2004; Ruiz-Pesini and Wallace 2006). However, subsequent studies have not supported

\footnotetext{
${ }^{4}$ Corresponding authors.

E-mail zhangyp@mail.kiz.ac.cn; fax 86-871-5195430.

E-mail ship@mail.kiz.ac.cn; fax 86-871-5199318.

Article published online before print. Article and publication date are at http:// www.genome.org/cgi/doi/10.1101/gr.093138.109.
}

this conclusion (Elson et al. 2004; Sun et al. 2007). Despite their focused controversies, these studies all acknowledged the role of selection in mtDNA evolution.

Mitochondrial oxidative phosphorylation (OXPHOS) generates not only heat but also adenosine triphosphate (ATP), which directly provides free energy for locomotion. Locomotion is energy-consuming, and flying birds (strongly locomotive birds) must require a more active metabolism than flightless birds (weakly locomotive birds). With this view, the mtDNA of weakly locomotive birds might have undergone relaxation of evolutionary constraints following the degeneration of locomotive ability. In contrast, strongly locomotive birds would have experienced stronger evolutionary constraints to eliminate deleterious mutations, and maintain efficient energy metabolism. As most nonsynonymous mutations are deleterious, they should be far less forgiving in strongly locomotive birds compared to weakly locomotive species.

Weakly locomotive birds would have lower dispersal ability, and many of them persist on islands only. Consequently, they likely have lower effective population sizes $(\mathrm{Ne})$. As $\mathrm{Ne}$ declines, slightly deleterious mutations have a greater probability of reaching fixation (Kimura 1962). Therefore, weakly locomotive birds likely accumulate more nonsynonymous mutations.

These observations regarding mitogenomic evolution lead to two questions. First, does the mtDNA of strongly and weakly locomotive birds accumulate different amounts of mutations? Second, what roles do $\mathrm{Ne}$ and functional constraints play in the evolution of mitochondrial genomes? To address these questions, we analyzed all avian mitochondrial genomes (71 species) available in GenBank. Because only 21 of the 71 species were flightless, we increased the database by sequencing four additional flightless 
and one flying species (Supplemental Table S1). If our hypothesis about birds is true, then we would expect to see a similar pattern in mammals (high- versus low-speed mammals). Thus, we further analyzed 214 complete mammalian mitochondrial genomes (Supplemental Table S2).

\section{Results and Discussion}

We reconstructed a maximum likelihood phylogenetic tree of birds based on 76 complete avian mitochondrial genomes (Fig. 1). The topology of this tree was very similar to that generated from a recent study based on 32 kilobases $(\mathrm{kb})$ of nuclear DNA sequence data (Hackett et al. 2008), although some internal nodes were ambiguous in our tree. As shown in Figure 1, flightless birds (marked in gray) did not cluster together.

Strength of selection is commonly measured by calculating the ratio of the rates of nonsynonymous (change in amino acid) substitution over synonymous (silent) substitutions $\left(K_{\mathrm{a}} / K_{\mathrm{s}}\right)$. To determine whether the mtDNA of different birds experienced differing selective pressures, we calculated the $K_{\mathrm{a}} / K_{\mathrm{s}}$ values associated with terminal branches (that is, $K_{\mathrm{a}} / K_{\mathrm{s}}$ since the most recent reconstructed ancestor) to measure the strength of selection during each species most recent divergence (see Methods).

To quantify the difference in $K_{\mathrm{a}} / K_{\mathrm{s}}$ ratios of birds with different locomotive ability, we first divided our samples into two groups: flightless and flying birds. Flightless birds referred to those having relatively small wing bones and lacking the ability to sustain flapping flight; they relied on running or swimming (Roots 2006). Poor fliers, those that can flap for a short distance, but are incapable of sustained flight (such as Meleagris gallopavo and the pheasants), were also classified into the flightless group. The mean $K_{\mathrm{a}} / K_{\mathrm{s}}$ ratio of all mtDNA genes was 0.050 in the flightless group, which is slightly greater (6.4\% larger) than that of the flying group. However, this dichotomy may not completely capture the difference in $K_{\mathrm{a}} / K_{\mathrm{s}}$ ratios because this did not consider the effects of energy consumption. Therefore, we also considered both classic behavioral and morphological characteristics, as well as features of
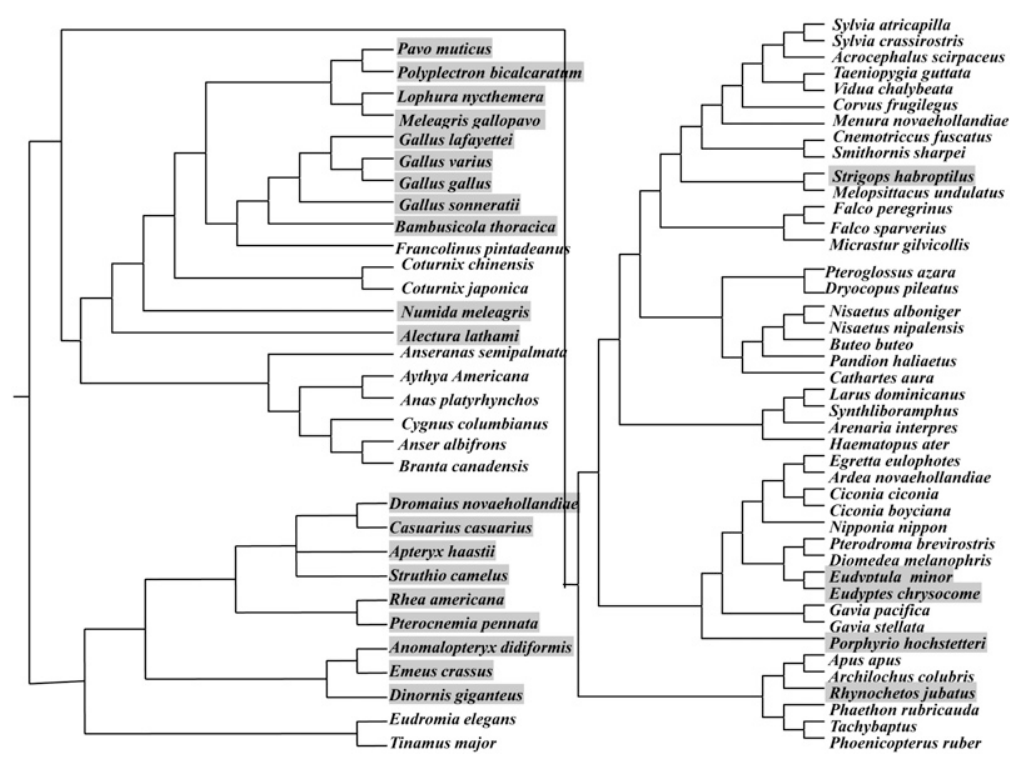

Figure 1. Avian phylogenetic tree reconstructed from mtDNA data. A phylogenetic tree of 76 avian species was constructed from all 13 protein-coding genes from mtDNA genomes using the maximum likelihood method. Flightless birds are marked in gray. energy consumption. The flying group included birds capable of sustained flight plus the poor flyers that can reach very high flight speeds, but do so rarely (e.g., Meleagris gallopavo), and fast runners (e.g., Casuarius casuarius, Rhea americana, and Struthio camelus) that can sustain speeds faster than the lowest speed of flyers (Patak and Baldwin 1993). This classification encapsulated almost all highenergy movement styles. These two groups were referred to as the weakly and strongly locomotive groups for convenience, respectively. With this classification, the difference of mean $K_{\mathrm{a}} / K_{\mathrm{s}}$ ratios increased from $6.4 \%$ to $16.5 \%$, indicating that relatively stronger negative selection occurred in the strongly locomotive group compared to the weakly locomotive group. There are rare speed records for weakly locomotive birds, thus it is possible that high-speed birds (without speed records) were involved in this group. But this should make our result more conservative because these high-speed birds should consume more energy, and then tend to elevate the average energy consumption level of the weakly locomotive group. Subsequently, we conducted a linear regression analysis comparing $K_{\mathrm{a}} / K_{\mathrm{s}}$ to the maximum speeds for 27 avian species. In general, the $K_{\mathrm{a}} / K_{\mathrm{s}}$ ratio was significantly negatively correlated with increasing speed $(P=0.016, R=0.459$; Fig. $2 \mathrm{~A})$, revealing a tendency for the $K_{\mathrm{a}} / K_{\mathrm{s}}$ ratio to decrease with flight speed.

Because weakly locomotive birds are distributed in different phylogenetic positions, our conclusions are not attributable to phylogenetic inertia. However, we used two methods to further explore for phylogenetic inertia. We compared 35 available, neighboring weakly-strongly locomotive pairs of birds (Supplemental Table S3) and found that the weakly locomotive birds had higher $K_{\mathrm{a}} / K_{\mathrm{s}}$ ratios in 32 of 35 pairs. Subsequently, phylogenetically independent contrasts (PIC) (Pickrell et al. 2009) analysis was used to overcome some of the potential phylogenetic biases, including variation in branch lengths (Ruiz-Pesini et al. 2004; Bjornerfeldt et al. 2006; Kivisild et al. 2006) and nonindependence of characters (locomotive speed). The PIC analyses showed the same decreasing trend in $K_{\mathrm{a}} / K_{\mathrm{s}}$ ratio with flight speed, although it was not significant $(P=0.109, R=0.717$; Fig. $2 \mathrm{~B})$. The nonsignificance of this result may have been due to the small sample size (six pairs) available for this test (Supplemental Table S4). Taken together, our results both from the general pattern and phylogenetic-based studies demonstrated that birds with weaker locomotive abilities accumulated more nonsynonymous mutations in the mtDNA than did those with stronger locomotive abilities.

Differences in mutation rate could have biased the results. The mean $K_{\mathrm{s}}$ $(0.441)$ of the strongly locomotive group was larger than that of the weakly locomotive group (0.330), although this difference was only marginally nearly insignificant $(P=0.059)$. The relatively higher $K_{\mathrm{s}}$ observed in the strongly locomotive group should have caused an underestimation of the $K_{\mathrm{a}} / K_{\mathrm{s}}$ ratio, and consequently favored our conclusion. To rectify this problem, we compared the mean $K_{\mathrm{a}}$ values between the weakly and strongly locomotive groups after controlling $K_{\mathrm{s}}$. Because all of the weakly 

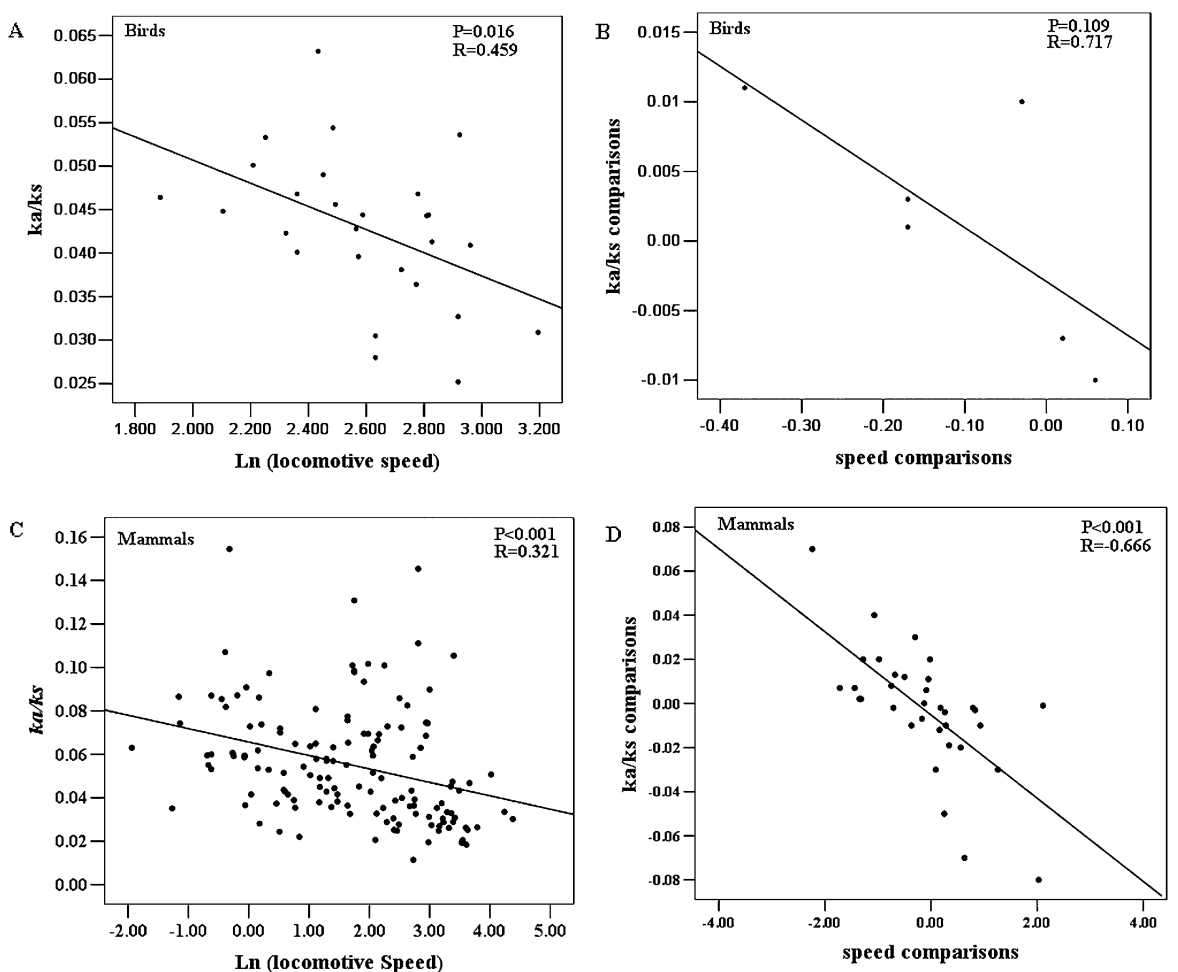

Figure 2. Negative correlation between mitochondrial $K_{\mathrm{a}} / K_{\mathrm{s}}$ ratios and locomotive speed. $(A)$ Ordinary linear regressions of $K_{\mathrm{a}} / K_{\mathrm{s}}$ ratios to loge speed for 27 avian species, raw cross-species values, $P=$ $0.016, R=0.459$. (B) Phylogenetically independent contrasts for six pairs of birds, $P=0.109, R=0.717$. (C) Ordinary linear regressions of $K_{\mathrm{a}} / K_{\mathrm{s}}$ ratios to loge speed for 131 mammalian species, raw crossspecies values, $P<0.001, R=0.321$. (D) Phylogenetically independent contrasts for 36 pairs of mammals, $P<0.001, R=-0.666$.

locomotive birds had clear phylogenetic positions with $K_{\mathrm{s}}$ values $<0.5$, we compared them with the 26 strongly locomotive birds that had $K_{\mathrm{s}}<0.5$. The mean $K_{\mathrm{s}}$ in the weakly locomotive group (0.250) was similar to that of the strongly locomotive group $(0.241$; $P=0.33)$. In contrast, the average $K_{\mathrm{a}}$ in the weakly locomotive group (0.013) was significantly greater than that of the strongly locomotive group $(0.009 ; P=0.035)$. Similarly, the mean $K_{\mathrm{a}} / K_{\mathrm{s}}$ ratio of the weakly locomotive group (0.05) was significantly larger than that of the strongly locomotive group $(0.04 ; P<0.01)$. The slightly greater $K_{\mathrm{s}}$ in the weakly locomotive group should lead to smaller $K_{\mathrm{a}} / K_{\mathrm{s}}$ ratios, thus making our result more conservative. Hence, these analyses revealed that the larger $K_{\mathrm{a}} / K_{\mathrm{s}}$ values in the weakly locomotive birds were not simply due to the differences in mutation rates $\left(K_{\mathrm{s}}\right)$.

Why do weakly locomotive birds have fixed more nonsynonymous mutations in their mtDNA? Most weakly locomotive birds, especially flightless birds, live on islands and have lower abilities to migrate. Thus, these birds tend to have smaller population sizes compared to strongly locomotive birds. Slightly deleterious nonsynonymous mutations are expected to accumulate faster in species with smaller effective population sizes, as negative selection is more effective in species with larger populations (Kimura 1962). Thus, differences in Ne of weakly and strongly locomotive birds could explain our observation. The most straightforward method to investigate the impact of $\mathrm{Ne}$ would be to compare the $K_{\mathrm{a}} / K_{\mathrm{s}}$ ratio between weakly and strongly locomotive birds with similar Ne. Unfortunately, it is not possible to achieve such comparisons owing to the limited amount of data on Ne.
However, it is possible to compare the $K_{\mathrm{a}} / K_{\mathrm{s}}$ ratio at other genomic loci between weakly and strongly locomotive birds. The influence of effective population size should affect all loci in the genome. Therefore, we compiled sequences for four nuclear genes (EGR1, BDNF, NGF, and NTF3) from 169 avian species (Supplemental Table S5) determined in a recent avian phylogenetic study (Hackett et al. 2008). Using the same criteria as above, after removing data where $K_{\mathrm{s}}=0$, the mean $K_{\mathrm{s}}$ was found to not differ significantly between the strongly $(0.037)$ and weakly $(0.042)$ locomotive birds $(P=$ 0.18 , Mann-Whitney $U$-test). In contrast, the mean $K_{\mathrm{a}}$ of the weakly locomotive group (0.0043) was marginally significantly greater than that of the strongly locomotive group $(0.0034 ; P=0.05)$, indicating that more nonsynonymous mutations have accumulated in the weakly locomotive group. These results are in accord with the Ne hypothesis. To compare the nuclear and mtDNA data sets, we repeated the above analysis using 20 species (four weakly locomotive birds and 16 strongly locomotive birds) that overlap in the two data sets. For the nuclear gene data set, $K_{\mathrm{a}}$ of the weakly locomotive group (0.003843) was greater than that of the strongly locomotive group (0.00298), whereas the $K_{\mathrm{s}}$ was not significantly different between the two groups $(P=0.17)$. A similar pattern was found for the mtDNA data set. Despite this, the $K_{\mathrm{a}} / K_{\mathrm{s}}$ ratio of the mtDNA genes in the weakly locomotive group was significantly larger than that in the strongly locomotive group $(P=0.008)$, whereas the ratio for nuclear genes was not significantly different between the two groups $(P=0.306)$. The Ne for mtDNA is $1 / 4$ of that for nuclear loci. Thus, mtDNA tends to accumulate more mutations and show elevated $K_{\mathrm{a}} / K_{\mathrm{s}}$. Due to this bias, we did not compare $K_{\mathrm{a}} / K_{\mathrm{s}}$ directly in two data sets, but rather compared the ratio of $K_{\mathrm{a}} / K_{\mathrm{s}}$ for the weakly versus strongly locomotive groups. The ratio of $K_{\mathrm{a}} / K_{\mathrm{s}}$ for the weakly versus strongly locomotive groups in the mtDNA data set was 1.28 , about $16 \%$ higher than that for the nuclear data set. These results suggested that the influence of $\mathrm{Ne}$ cannot fully explain our finding of an enhancement of $K_{\mathrm{a}} / K_{\mathrm{s}}$ in weakly locomotive birds.

An alternative hypothesis to explain our finding is that the functional constraints were relaxed on the mtDNA in weakly locomotive birds following the degeneration of locomotive ability. The metabolic power required for locomotion is linearly related to speed (Taylor 1982). Thus, if our hypothesis of functional constraints on mtDNA is true, then maximum speeds would be expected to correlate with the $K_{\mathrm{a}} / K_{\mathrm{s}}$ ratio of mtDNA genes, but not for nuclear genes. Consequently, we performed linear regression analysis comparing $K_{\mathrm{a}} / K_{\mathrm{s}}$ to maximum speeds for nine avian species where we had $K_{\mathrm{a}} / K_{\mathrm{s}}$ values for mtDNA and nuclear genes, as well as data on maximum locomotive speeds. As expected, the $K_{\mathrm{a}} / K_{\mathrm{s}}$ ratio for mtDNA has a significant negative correlation with increasing maximum speed $\left(P=0.036, R^{2}=0.344\right)$. In contrast, the $K_{\mathrm{a}} / K_{\mathrm{s}}$ ratio of nuclear genes was not significantly negatively 
correlated to increasing speed $\left(P=0.508, R^{2}=0.041\right)$. Moreover, the significant linear relationship was not due to changes in $K_{\mathrm{s}}$ because we failed to find a significant linear relationship between $K_{\mathrm{s}}$ and maximum speed $\left(P=0.23, R^{2}=0.08\right)$. In contrast, the relationship was most likely due to the increase of $K_{\mathrm{a}}\left(R^{2}=0.28\right)$, although it was only nearly significant $(P=0.09)$. These observations were consistent with the hypothesis of the relaxation of functional constraints.

Proteins encoded by mtDNA play a critical role in OXPHOS, providing up to $95 \%$ of the cell's energy requirements. Because nonsynonymous substitutions tend to be more harmful than synonymous substitutions, strongly locomotive birds experience stronger evolutionary constraints on their mtDNA than do weakly locomotive birds. Nonsynonymous (deleterious) mutations are purified through selection to maintain an efficient energy generation system. A caveat of this analysis is that, due to data limitations, only nine species whose mtDNA, nuclear genes, and speed data are all available. Additional species would be helpful to verify our results.

For many proteins, selection results in nonrandom radical amino acid substitutions (Zhang 2000). To investigate whether this is the case for our data set, we investigated the pattern of conservative and radical nonsynonymous substitutions. Here, we compared four types of radical amino acid changes: polarity-based $K_{\mathrm{r}} / K_{\mathrm{c}}$, volume-based $K_{\mathrm{r}} / K_{\mathrm{c}}$, polarity-volume-based $K_{\mathrm{r}} / K_{\mathrm{c}}$, and chargebased $K_{\mathrm{r}} / K_{\mathrm{c}}$. In three of the four analyses, the $K_{\mathrm{r}} / K_{\mathrm{c}}$ ratio (polarity, volume, and polarity-volume) showed a significant relationship with maximum speed in birds. The charge-based $K_{\mathrm{r}} / K_{\mathrm{c}}$ was the only molecular trait that did not significantly differ between strongly versus weakly locomotive birds. This may be due to the stronger purifying selection against changes in amino acid charge for the proteins under study (Popadin et al. 2007). Consequently, these results further support the notion that stronger selective constraints are acting on strongly locomotive birds.

We investigated the general pattern for the combined 13 protein-coding genes from the mtDNA genome, but did all of the mtDNAencoding genes experience the same selection? For an analysis of the individual mitochondrial protein-coding genes, we repeated the above analysis for each of the 13 individual genes and for sets of genes involved in OXPHOS complexes $I$, $I V$, and $V$ (Supplemental Table S7). The $K_{\mathrm{a}} / K_{\mathrm{s}}$ ratios varied among the different genes, implying that different genes accumulated different amounts of deleterious mutations (Fig. 3). Only ND1, ND6, COXIII, ATP6, and complexes $I$ and $V$ showed significant differences between the strongly and weakly locomotive groups, while the other genes and complex $I V$ did not show any significant differences. In OXPHOS, some genes may play more important roles than other genes. Therefore, it is reasonable to assume that some genes have undergone stronger selective constraints to eliminate deleterious mutations to maintain their function than others.

Because functional constraints influence the evolution of mtDNA in birds, we hypothesized that mammals with differing locomotive speeds also exhibit a similar pattern. The larger quantity of mammalian data should verify our results obtained from the smaller avian sample. We compiled 214 mammalian mitochondrial genomes and classified the species into two groups based on their maximum relative speed. This comparison of locomotive performance must consider the effect of body length, as both the extent of muscle contraction and the distance of onestep traveled vary directly with body length. Maximum relative speed, measured by the maximum speed controlled for body length, was suggested to be a more appropriate proxy for locomotive performance (Jones and Lindstedt 1993; Iriarte-Diaz 2002). High-speed mammals had an $\operatorname{Ln} S>1.79$ (where 1.79 was the median of loge-transformed speed $S$ ), and low-speed mammals had an $\operatorname{Ln} S<1.79$ (Supplemental Table S2). The $K_{\mathrm{a}} / K_{\mathrm{s}}$ ratio was found to be significantly smaller in high-speed mammals compared to low-speed mammals $(P=0.005)$. In addition, linear regression $(P<$ $0.001, R=0.321$; Fig. 2C) and phylogenetically independent contrasts $(P<0.001, R=-0.666$; Fig. 2D; Supplemental Table S6) showed significant negative relationships between the $K_{\mathrm{a}} / K_{\mathrm{s}}$ ratio and locomotive speed. These findings strengthen our conclusion from birds; the mtDNA of strongly locomotive animals has undergone stronger evolutionary constraints.

Strongly locomotive animals (birds and mammals) have lower $K_{\mathrm{a}} / K_{\mathrm{s}}$ ratios in their mtDNA than do weakly locomotive species. Indeed, strongly locomotive species accumulate fewer nonsynonymous mutations. Nonsynonymous substitutions are generally more harmful than synonymous substitutions, as they can cause defects in respiratory-chain activity that reduce the efficiency of skeletal muscle (Weber et al. 1997; Brown et al. 2000), as well as other metabolic processes (Taylor and Turnbull 2005; Wallace 2005). Thus, the mtDNA of strongly locomotive species has experienced stronger purifying selection to maintain efficient energy metabolism. In weakly locomotive species, due to their lower demand for energy, individuals that have lower metabolic efficiency are more likely to survive and reproduce in a population

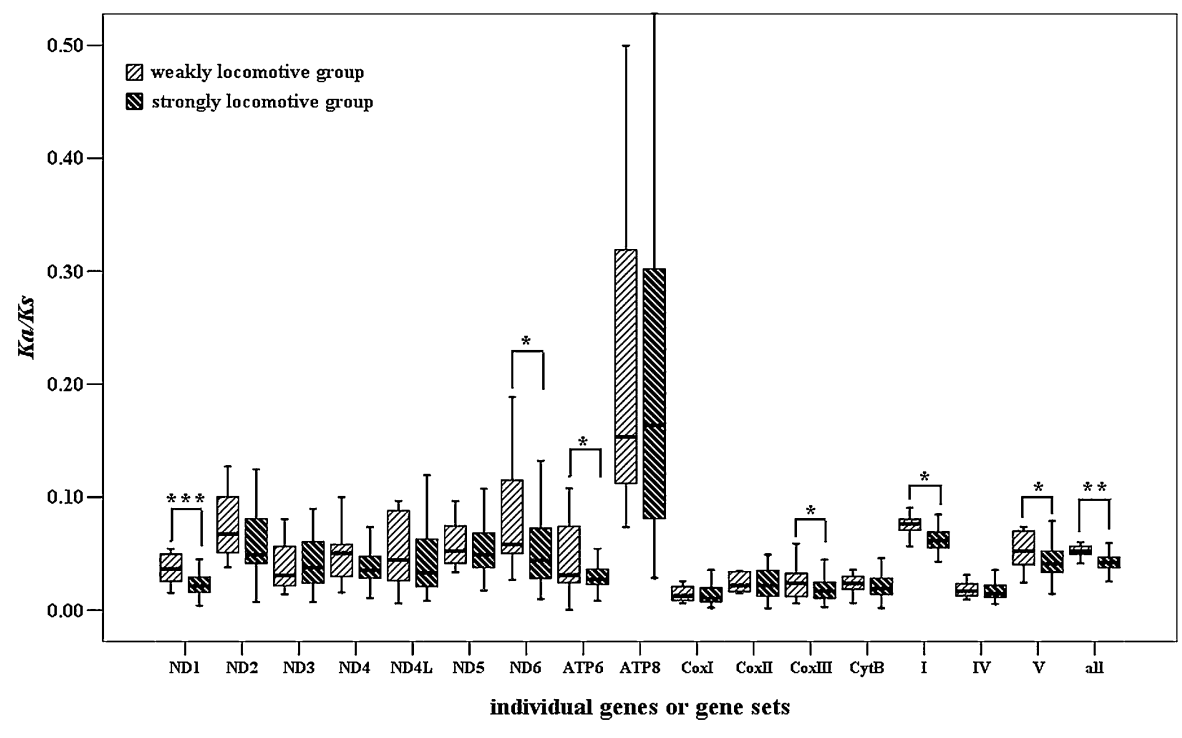

Figure 3. Comparisons of average $K_{\mathrm{a}} / K_{\mathrm{s}}$ ratios for 13 individual genes, genes involved in complexes $I_{\text {, }}$ $I V$, and $V$, and 13 protein-coding genes set between weakly and strongly locomotive groups. Boxes include $50 \%$ of the distributions. ND1, $P<0.001 ; N D 2, P>0.05 ; N D 3, P>0.05 ; N D 4, P>0.05 ; N D 4 L$, $P>0.05 ;$ ND5, $P>0.05 ;$ ND6, $P<0.05 ;$ ATP6, $P<0.05 ;$ ATP8, $P>0.05 ;$ COXI, $P>0.05 ;$ COXII, $P>0.05$; COXIII, $P<0.05$; CytB, $P>0.05$; complex $I, P<0.05$; complex IV, $P>0.05$; complex $V, P<0.05$; all genes, $P<0.001$. Note: ${ }^{*} P<0.05,{ }^{* *} P=0.05-0.001,{ }^{* * *} P<0.001$. 
than strongly locomotive species under similar circumstances. The relaxation of metabolic constraint may allow for the accumulation of additional nonsynonymous mutations in the mitochondrial genome (Bjornerfeldt et al. 2006). In conclusion, differing constraints on energy metabolism, in addition to the influence of effective population size, explain differences in selective patterns in mtDNA of animals with different locomotive abilities.

\section{Methods}

\section{Source of data and primary treatment}

Raw mitochondrial genomes from all 71 bird species available in January 2008 were downloaded from GenBank. Mitochondrial genomes for five additional species were sequenced for this study (GenBank accession numbers EU165706, EU165707, and EU417810-417812; Supplemental Table S1). The LA-PCR primer sets and segmental amplification primer sets were used as described by Nishibori et al. (2001), with 14 additional oligonucleotide primers designed for this study. The PCR products were sequenced at least three times on an ABI 3730 Sequencer (Applied Biosystems) using the ABI PRISM BigDye Terminator v3.0. All 13 protein-coding mitochondrial genes were extracted from each genome and translated into amino acid sequences.

Likewise, raw mitochondrial genomes from 214 mammalian species available in August 2007 were downloaded from GenBank. The 13 protein-encoding mitochondrial genes were extracted from each genome and translated into amino acid sequences. Amino acid sequences for each gene were aligned by ClustalX 1.81 (Thompson et al. 1997) with default settings, verified by visual inspection, and used as guides to align the nucleotide sequences.

In addition, four nuclear genes ( $E G R 1, B D N F, N G F$, and NTF3) for 169 species of birds were collected from a recent avian phylogenetic study (Hackett et al. 2008).

Data for maximum speed (locomotive speed) and body length for each species were collected from previous studies (Garland 1983; Christiansen 2002; Iriarte-Diaz 2002; Alerstam et al. 2007) and the "Animal Diversity Web" (Myers et al. 2006; Table 2; Supplemental Table S1). For flying birds, as soaring flight is less energyintensive than flapping flight, in this study, we used the maximum flapping-flight speed to represent maximum active energy. For mammals, comparison of locomotive performance among taxa suffers from the problem of scale because body size affects nearly all physiological functions. Because the amount that an animal's muscle shortens, and the distance traveled with one step, both vary directly with body length, a body length-dependent scale (relative running speed) might be more appropriate when characterizing the performance of animals (Jones and Lindstedt 1993; Iriarte-Diaz 2002). Therefore, in this study, we used "relative speed" (maximal speed divided by body length) to standardize speed among taxa.

\section{Phylogenetic analysis}

Concatenated amino acid sequences of all 13 protein-coding genes were used to construct a maximum likelihood phylogenetic tree for birds and mammals using PhyML (Guindon and Gascuel 2003). The avian phylogenetic tree based on 13 protein-coding genes showed relationships similar to that from a recent study based on 19 nuclear genes that yielded a robust avian phylogenetic tree (Hackett et al. 2008). We used their robust nuclear tree to guide our mitochondrial phylogeny (Fig. 1). For those species with low bootstrap in our tree, while they were highly supported in Hackett's phylogeny (Hackett et al. 2008), we used their topology. And these species were marked in Supplemental Table S1.

\section{Ratio of nonsynonymous to synonymous nucleotide substitutions $\left(K_{\mathrm{a}} / K_{\mathrm{s}}\right)$}

The ratio of the rates of nonsynonymous to synonymous substitutions $\left(K_{\mathrm{a}} / K_{\mathrm{s}}\right)$ of all individual data sets were estimated for each branch of the phylogenetic tree using PAML (Yang 1997, 2007). Because the independent estimation of the $K_{\mathrm{a}} / K_{\mathrm{s}}$ ratios for each branch of the tree was extremely time-consuming, the mammalian tree was divided into nine monophyletic subtrees, while the avian tree was divided into three subtrees, each of which was evaluated independently. For each subtree, model 1 with a free $K_{\mathrm{a}} / K_{\mathrm{s}}$ ratio was estimated separately for each branch. Only $K_{\mathrm{a}} / K_{\mathrm{s}}$ values associated with terminal branches were used in the subsequent analyses. Thus, we focused only on the rate of accumulation of slightly deleterious mutations $\left(K_{\mathrm{a}} / K_{\mathrm{s}}\right.$ and $\left.K_{\mathrm{r}} / K_{\mathrm{c}}\right)$ between modern species and their most recent reconstructed ancestors.

\section{Ratio of radical to conservative amino acid substitutions $\left(K_{\mathrm{r}} / K_{\mathrm{c}}\right)$}

The ratio of the rates of radical-to-conservative substitutions $\left(K_{\mathrm{r}} / K_{\mathrm{c}}\right)$ of the concatenated 13 protein-encoding genes was estimated by comparing the nucleotide sequences of modern animals to those of their most recent reconstructed ancestors using Zhang's algorithm (Zhang 2000). Ancestral nucleotide sequences were constructed with PAML (Yang 1997, 2007). The Jukes-Cantor model was used to correct for multiple hits, as $K_{\mathrm{r}}$ and $K_{\mathrm{c}}$ values were very small $(<0.3)$. The 20 amino acids were classified into two groups in four different ways according to their (1) charge, (2) polarity, (3) volume, and (4) both polarity and volume (Zhang 2000; Popadin et al. 2007).

\section{Statistical treatments}

As the study of Popadin et al. (2007) suggested that effective population size is the major factor influencing the fixation of deleterious mutations in mtDNA. In order to compare our results to theirs, the same statistical analyses were used. In brief, we compared the average values of the molecular traits $\left(K_{\mathrm{a}} / K_{\mathrm{s}}, K_{\mathrm{r}} / K_{\mathrm{c}}\right.$, $K_{\mathrm{s}}$, and $K_{\mathrm{c}}$ ), which belonged to the weak versus high locomotive groups. Subsequently, we performed an ordinary linear regressions analysis of the molecular traits against speed. Selection strength $\left(K_{\mathrm{a}} / K_{\mathrm{s}}\right.$ and $\left.K_{\mathrm{r}} / K_{\mathrm{c}}\right)$ was considered to be the dependent variable, while Ln $S$ (loge-transformed speed $S$ ) was considered to be the independent variable.

Phylogenetic inertia (closely related species tend to be similar because of shared inheritance, rather than through independent adaptation), might compromise comparative species analyses (Harvey and Pagel 1991; Fisher and Owens 2004). The characters we analyzed may not be independent among species. Thus, ordinary statistics, such as correlation and regression, may overstate significance in hypothesis testing. Thus, in addition, we also used phylogenetically independent contrasts (Felsenstein 1985; Pagel 1992), which was implemented in the program COMPARE 4.6b (Martins 2004) to overcome the problem of nonindependence.

\section{Acknowledgments}

Special thanks to Dr. David Irwin, Dr. R.W. Murphy, Scott Groom, Jing He, and two anonymous reviewers for helpful comments. This work was supported by grants from National Basic Research Program of China (973 Program, 2007CB411600), National Natural Science Foundation of China (30621092), and Bureau of Science and Technology of Yunnan Province to Y.-P.Z., and by a start-up fund from the "Hundreds-Talent Program" of the Chinese Academy of Sciences to P.S.

\section{Genome Research}

www.genome.org 


\section{References}

Alerstam T, Rosén M, Bäckman J, Ericson PGP, Hellgren O. 2007. Flight speeds among bird species: Allometric and phylogenetic effects. PLoS Biol 5: e197. doi: 10.1371/journal.pbio.0050197.

Ballard JWO, Whitlock MC. 2004. The incomplete natural history of mitochondria. Mol Ecol 13: 729-744.

Berg C, Rayner J. 1995. The moment of inertia of bird wings and the inertial power requirement for flapping flight. J Exp Biol 198: 1655-1664.

Bjornerfeldt S, Webster MT, Vila C. 2006. Relaxation of selective constraint on dog mitochondrial DNA following domestication. Genome Res 16: 990-994.

Brown MD, Trounce IA, Jun AS, Allen JC, Wallace DC. 2000. Functional analysis of lymphoblast and cybrid mitochondria containing the 3460 , 11778 , or 14484 Leber's hereditary optic neuropathy mitochondria DNA mutation. J Biol Chem 275: 39831-39836.

Castoe TA, Jiang ZJ, Gu W, Wang ZO, Pollock DD. 2008. Adaptive evolution and functional redesign of core metabolic proteins in snakes. PLoS One 3: e2201. doi: 10.1371/journal.pone.0002201.

Chiappe LM. 2007. Glorified dinosaurs: The origin and early evolution of birds. Wiley, Hoboken, NJ.

Christiansen PER. 2002. Locomotion in terrestrial mammals: The influence of body mass, limb length and bone proportions on speed. Zool J Linn Soc 136: $685-714$.

da Fonseca RR, Johnson WE, O'Brien SJ, Ramos MJ, Antunes A. 2008. The adaptive evolution of the mammalian mitochondrial genome. $B M C$ Genomics 9: 119. doi: 10.1186/1471-2164-9-119.

Elson JL, Turnbull DM, Howell N. 2004. Comparative genomics and the evolution of human mitochondrial DNA: Assessing the effects of selection. Am J Hum Genet 74: 229-238.

Felsenstein J. 1985. Phylogenies and the comparative method. Am Nat 125: $1-15$.

Fisher DO, Owens IPF. 2004. The comparative method in conservation biology. Trends Ecol Evol 19: 391-398.

Garland T. 1983. The relation between maximal running speed and body mass in terrestrial mammals. J Zool 199: 157-170.

Guindon S, Gascuel O. 2003. A simple, fast, and accurate algorithm to estimate large phylogenies by maximum likelihood. Syst Biol 52: 696-704.

Hackett SJ, Kimball RT, Reddy S, Bowie RCK, Braun EL, Braun MJ, Chojnowski JL, Cox WA, Han KL, Harshman J, et al. 2008. A phylogenomic study of birds reveals their evolutionary history. Science 320: $1763-1768$.

Harvey D, Pagel M. 1991. The comparative method in evolutionary biology. Oxford University Press, Oxford, UK.

Iriarte-Diaz J. 2002. Differential scaling of locomotor performance in small and large terrestrial mammals. J Exp Biol 205: 2897-2908.

Jones JH, Lindstedt SL. 1993. Limits to maximal performance. Annu Rev Physiol 55: 547-569.

Kimura M. 1962. On the probability of fixation of mutant genes in a population. Genetics 47: 713-719.

Kivisild T, Shen P, Wall DP, Do B, Sung R, Davis K, Passarino G, Underhill PA, Scharfe C, Torroni A, et al. 2006. The role of selection in the evolution of human mitochondrial genomes. Genetics 172: 373-387.

Martins E. 2004. Compare, version 4.6b. Computer programs for the statistical analysis of comparative data. Indiana University, Bloomington, IN.

Mishmar D, Ruiz-Pesini E, Golik P, Macaulay V, Clark AG. 2003. Natural selection shaped regional mtDNA variation in humans. Proc Natl Acad Sci 100: 171-176.
Myers P, Espinosa R, Parr CS, Jones T, Hammond GS, Dewey TA. 2006. The animal diversity web. http://animaldiversity.org.

Nishibori M, Hayashi T, Tsudzuki M, Yamamoto Y, Yasue H. 2001. Complete sequence of the Japanese quail (Coturnix japonica) mitochondrial genome and its genetic relationship with related species. Anim Genet 32: 380-385.

Pagel MD. 1992. A method for the analysis of comparative data. J Theor Biol 156: 431-442.

Patak A, Baldwin J. 1993. Structural and metabolic characterisation of the muscles used to power running in the emu (Dromaius novaehollandiae), a giant flightless bird. J Exp Biol 175: 233-249.

Pickrell JK, Coop G, Novembre J, Kudaravalli S, Li JZ, Absher D, Srinivasan BS, Barsh GS, Myers RM, Feldman MW, et al. 2009. Signals of recent positive selection in a worldwide sample of human populations. Genome Res 19: 826-837.

Popadin K, Polishchuk LV, Mamirova L, Knorre D, Gunbin K. 2007. Accumulation of slightly deleterious mutations in mitochondrial protein-coding genes of large versus small mammals. Proc Natl Acad Sci 104: $13390-13395$.

Rand, D.M. 2008. Mitigating mutational meltdown in mammalian mitochondria. PLOS Biol 6: e35. doi: 10.1371/journal.pbio.0060035.

Roots C. 2006. Flightless birds. Greenwood Press, Santa Barbara, CA.

Ruiz-Pesini E, Wallace DC. 2006. Evidence for adaptive selection acting on the tRNA and rRNA genes of human mitochondrial DNA. Hum Mutat 27: 1072-1081.

Ruiz-Pesini E, Mishmar D, Brandon M, Procaccio V, Wallace DC. 2004. Effects of purifying and adaptive selection on regional variation in human mtDNA. Science 303: 223-226.

Sibley CG, Ahlquist JE. 1990. Phylogeny and classification of birds. Yale University Press, New Haven, CT

Sun C, Kong QP, Zhang YP. 2007. The role of climate in human mitochondrial DNA evolution: A reappraisal. Genomics 89: 338-342.

Taylor CR. 1982. Energetics and mechanics of terrestrial locomotion. I. Metabolic energy consumption as a function of speed and body size in birds and mammals. J Exp Biol 97: 1-21.

Taylor RW, Turnbull DM. 2005. Mitochondrial DNA mutations in human disease. Nat Rev Genet 6: 389-402.

Thompson JD, Gibson TJ, Plewniak F, Jeanmougin F, Higgins DG. 1997. The clustal_x windows interface: Flexible strategies for multiple sequence alignment aided by quality analysis tools. Nucleic Acids Res 25: $4876-4882$.

Wallace DC. 2005. A mitochondrial paradigm of metabolic and degenerative diseases, aging, and cancer: A dawn for evolutionary medicine. Annu Rev Genet 39: 359-407.

Weber K, Wilson JN, Taylor L, Brierley E, Johnson MA, Turnbull DM, Bindoff LA. 1997. A new mtDNA mutation showing accumulation with time and restriction to skeletal muscle. Am J Hum Genet 60: 373-380.

Withers PC. 1981. An aerodynamic analysis of bird wings as fixed aerofoils. J Exp Biol 90: 143-162.

Yang ZH. 1997. PAML: A program package for phylogenetic analysis by maximum likelihood. Comput Appl Biosci 13: 555-556.

Yang Z. 2007. PAML 4: Phylogenetic analysis by maximum likelihood. Mol Biol Evol 24: 1586-1591.

Zhang J. 2000. Rates of conservative and radical nonsynonymous nucleotide substitutions in mammalian nuclear genes. J Mol Evol 50: 56-68.

Received February 15, 2009; accepted in revised form June 16, 2009. 


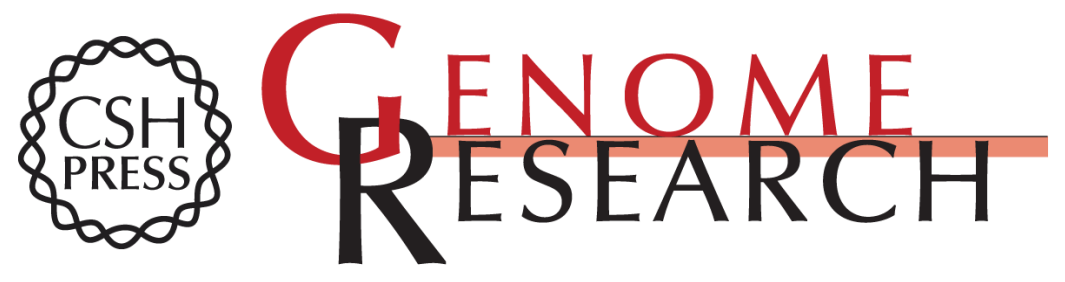

\section{Relaxation of selective constraints on avian mitochondrial DNA following the degeneration of flight ability}

Yong-Yi Shen, Peng Shi, Yan-Bo Sun, et al.

Genome Res. 2009 19: 1760-1765 originally published online July 17, 2009

Access the most recent version at doi:10.1101/gr.093138.109

Supplemental Material

References

License

Email Alerting Service
http://genome.cshlp.org/content/suppl/2009/09/02/gr.093138.109.DC1

This article cites 37 articles, 13 of which can be accessed free at: http://genome.cshlp.org/content/19/10/1760.full.html\#ref-list-1

Receive free email alerts when new articles cite this article - sign up in the box at the top right corner of the article or click here.

\section{Affordable, Accurate Sequencing.}

